\title{
Synthesis and primary antiviral activity evaluation of 3-hydrazono-5-nitro-2-indolinone derivatives
}

\author{
Nalan Terzioğlu *a, Nilgün Karalı ${ }^{\text {a }}$, Aysel Gürsoy ${ }^{\text {a }}$, Christophe Pannecouque ${ }^{\text {b, }}$ \\ Pieter Leysen $^{\text {b }}$, Jan Paeshuyse ${ }^{\text {b }}$, Johan Neyts ${ }^{\text {b }}$, and Erik De Clercq ${ }^{\text {b }}$ \\ ${ }^{a}$ Istanbul University, Faculty of Pharmacy, Department of Pharmaceutical Chemistry, 34116, \\ Istanbul, Turkey \\ ${ }^{b}$ Rega Institue of Medical Research, Katholieke Universiteit Leuven, B-3000 Leuven, Belgium \\ E-mail:nalant@yahoo.com
}

\begin{abstract}
A series of 5-nitro-3-[(5-nonsubstituted/methyl-4-thiazolidinone-2-ylidene)hydrazono]-1H-2indolinones (3a-j and 4a-h) was synthesized by cyclization of 5-nitro-1H-indole-2,3-dione-3thiosemicarbazones (1a-k) with ethyl bromoacetate or ethyl 2-bromopropionate. The primary antiviral activities of the new hydrazonoindolinone derivatives, the previously reported 5-nitro$1 \mathrm{H}$-indole-2,3-dione-3-thiosemicarbazones (1a-k) and 1-morpholino/piperidinomethyl-5-nitroindole-2,3-dione-3-thiosemicarbazones (2a-l) were evaluated against some pathogenic viruses in the Rega Institue for Medical Research, Belgium. Among the tested compounds, 1c, $\mathbf{2 b}$ and $\mathbf{3 b}$ afforded some weak activity against the yellow fever virus (YFV) in vero cells, whereas compounds $\mathbf{2 b}, \mathbf{3 a}, \mathbf{3 f}, \mathbf{4 e}$ and $\mathbf{4 f}$ inhibited the growth of bovine viral diarrhea virus (BVDV) in MDBK CODA cells.
\end{abstract}

Keywords: $1 H$-2-Indolinones, 4-thiazolidinones, antiviral activity

\section{Introduction}

Isatin ( $1 H$-indole-2,3-dione) is a versatile lead molecule for designing potential antiviral agents. Several authors found that isatin- $\beta$-thiosemicarbazone (1H-indole-2,3-dione-3thiosemicarbazone) and its N-Mannich bases were active against various viruses ${ }^{1}$. The first clinically approved antiviral agent, $\mathrm{N}$-methylisatin- $\beta$-thiosemicarbazone (methisazone, I), and isatin- $\beta$-thiosemicarbazone (II) are active against poxviruses ${ }^{2}$. Antiviral properties of certain thiourea and semicarbazone derivatives have been reported in which the antiviral effect is attributed to the presence of an intact $\mathrm{NHC}(=\mathrm{S}) \mathrm{NH}$ grouping and $\mathrm{NHC}(=\mathrm{O}) \mathrm{NH}$ grouping ${ }^{3}$. The cyclic urea derivative (III), as a replicase inhibitor specific to the bovine viral diarrhea virus $(\mathrm{BVDV})^{4}$ has been recently identified. On the other hand, some original 4-thiazolidones are 
undergoing different stages of clinical trials as potential antimicrobial, antiviral, anticancer and thrombolytic drugs. It has been reported that members of a series of 2,3-diaryl-1,3-thiazolidin-4ones were highly effective in inhibiting the cytopathic effect of HIV-1 in human T-lymphocyte cells ${ }^{5}$. The 2-thioxo-4-thiazolidone derivative, epalrestat (IV) is a highly active aldose reductase inhibitor (Figure 1).

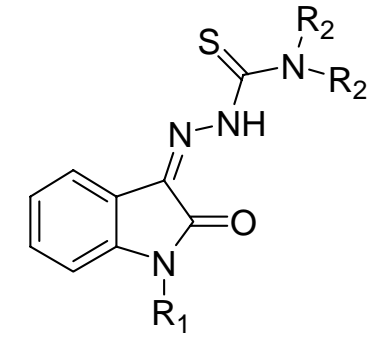

I $\mathrm{R}_{1}=\mathrm{CH}_{3}, \quad \mathrm{R}_{2}=\mathrm{H}$
II $\mathrm{R}_{1}=\mathrm{R}_{2}=\mathrm{H}$<smiles>CCN(CC)CCn1c(=O)[nH]c2ccc(-n3ccnc3)cc21</smiles>

III<smiles>CC(/C=C1\SC(=S)N(CC(=O)O)C1=O)=C\c1ccccc1</smiles>

IV

\section{Figure 1}

In view of these observations, we report here the synthesis of the new 5-nitro-3-[(5nonsubstituted/ methyl-4-thiazolidinone-2-ylidene)hydrazono]-1H-2-indolinones (3 and 4) and evaluation of in vitro antiviral activity of these new compounds and some of the previously reported 5-nitro-1H-indole-2,3-dione-3-thiosemicarbazones (1) and 1-morpholino/ piperidinomethyl-5-nitro-indole-2,3-dione-3-thiosemicarbazones (2).

\section{Results and Discussion}

Treatment of ethyl bromoacetate or ethyl 2-bromopropionate with 5-nitro-1H-indole-2,3-dione3-thiosemicarbazone 1a-k in anhydrous ethanolic medium furnished the corresponding 5-nitro-3[(5-nonsubstituted/methyl-4-thiazolidinone-2-ylidene)hydrazono]-1H-2-indolinones (3a-j and 4a-h) (Scheme 1).

The structures of $\mathbf{3}$ and $\mathbf{4}$ were confirmed by analytical and spectral data (IR, ${ }^{1} \mathrm{H} \mathrm{NMR},{ }^{13} \mathrm{C}$ NMR and EIMS) (Table 1). In the IR spectra of $\mathbf{3}$ and $\mathbf{4}$, the $\mathrm{C}=\mathrm{S}$ bands disappeared, a new $\mathrm{C}=\mathrm{O}$ band (1756-1734 $\left.\mathrm{cm}^{-1}\right)$ indicative of the thiazolidinone structure appeared in addition to the lactam $\mathrm{C}=\mathrm{O}$ stretching $\left(1705-1689 \mathrm{~cm}^{-1}\right)^{6} .{ }^{1} \mathrm{H}-\mathrm{NMR}$ spectra of $\mathbf{3}$ and $\mathbf{4}$ did not display the signals of the thiosemicarbazone protons and exhibited a singlet or two singlets resulting from the indole 
$\mathrm{NH}(\delta 11.28-11.43 \mathrm{ppm})$. The $\mathrm{SCH}_{2}$ protons of 3 were observed as two singlets $(\delta$ 4.09-4.20 ppm and 4.11-4.24 ppm) due to anisotropic effect of carbonyl group. The $\mathrm{CH}_{3}(\delta 1.60-1.72 \mathrm{ppm})$ and the $\mathrm{SCH}(\delta 4.47-4.58 \mathrm{ppm})$ protons of 4 resonated as a doublet and a quartet, respectively ${ }^{7}$. In EIMS spectrum, 3d, 3f, 3i, 4c and 4e chosen as prototypes showed molecular ions with different intensity and the fragments peculiar to the indole and thiazolidinone moieties ${ }^{8}$.

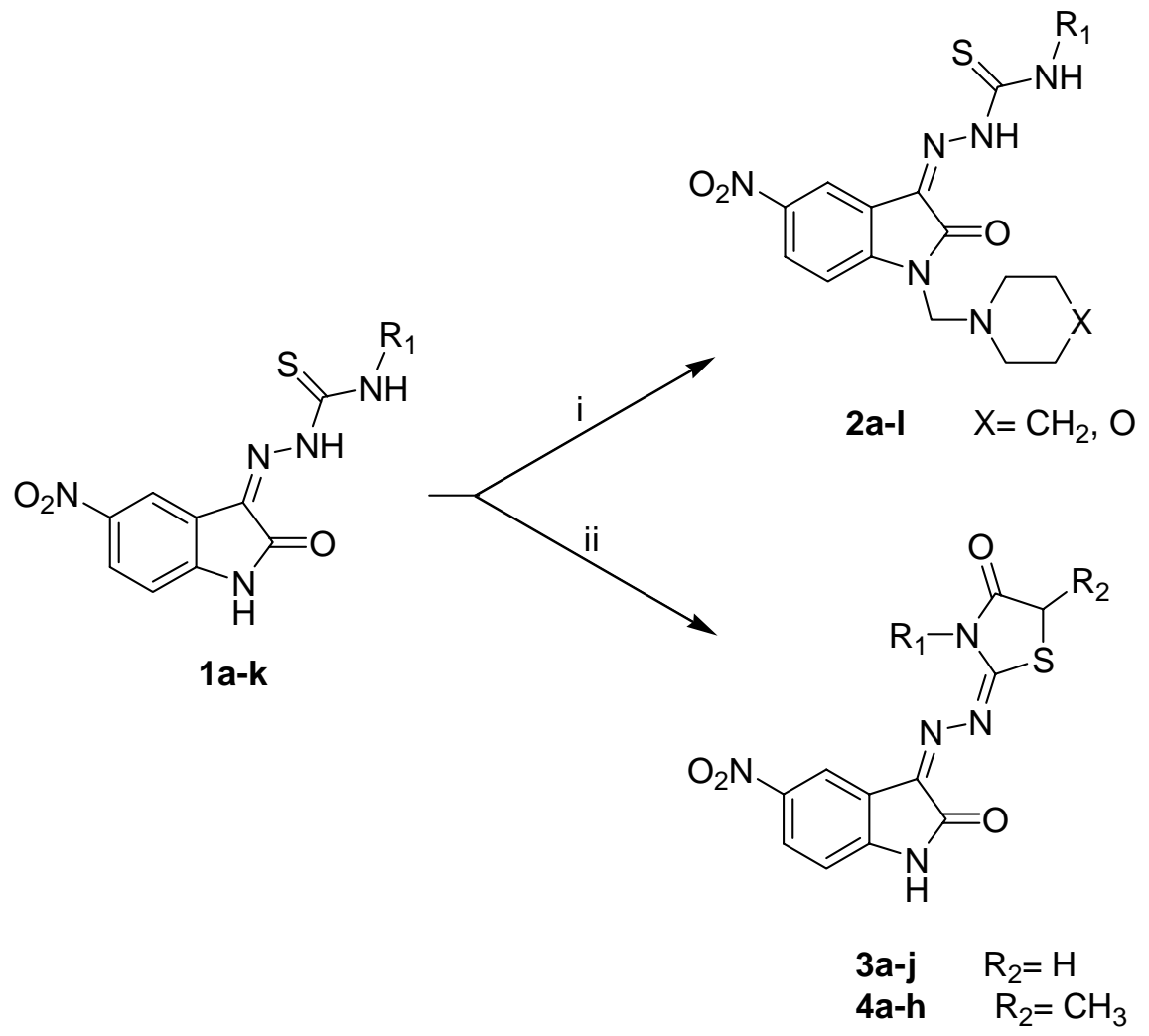

Scheme 1. Reagents and conditions: (i) piperidine/morpholine, HCHO, EtOH (ii) ethyl bromoacetate / ethyl 2-bromopropionate, anhyd. $\mathrm{CH}_{3} \mathrm{COONa}$, EtOH.

The new 3-hydrazono-5-nitro-2-indolinone derivatives 3a-j and 4a-h and the previously synthesized $^{9}$ 5-nitro-1H-indol-2,3-dion-3-thiosemicarbazones 1a-k and N-Mannich bases 2a-l were evaluated for in vitro antiviral activity against the yellow fever virus (YFV) (strain 17D) in vero cells and the bovine viral diarrhea virus (BVDV-strain NADL) in Madin-Dardy bovine kidney (MDBK CODA) cells in Rega Institue for Medical Research, Belgium ${ }^{4,10,11}$. For each compound, the $50 \%$ effective concentration $\left(\mathrm{EC}_{50}\right)$ and the minimal toxic concentration (MTC) or the $50 \%$ cytotoxic concentration $\left(\mathrm{CC}_{50}\right)$ were obtained. Ribavirin (Rib) was used as the standard in the tests. Both antiviral activity and cytotoxicity were determined by means of the MTS method. As can be seen in Table 2, some of the tested compounds afforded some weak activity against YFV, but at subtoxic concentrations. Among the compounds tested, the MTC 
value of $\mathbf{1 c}, \mathbf{2 b}$ and $\mathbf{3 b}$ are $100 \mu \mathrm{g} / \mathrm{mL}$ and the $\mathrm{EC}_{50}$ of these compounds are 2.18, 11 and 2.52 $\mu \mathrm{g} / \mathrm{mL}$, respectively. The $\mathrm{EC}_{50}$ and the MTC values of ribavirin are 45 and $>100 \mu \mathrm{g} / \mathrm{mL}$ in the tests against YFV, respectively. When these data are examined, it is observed that $\mathbf{1 c}, \mathbf{2 b}$ and $\mathbf{3 b}$ are more active, but more toxic than ribavirin. The most active compound against YFV was $\mathrm{R}_{1}$ allyl substituted thiosemicarbazone derivative 1c. In comparison with 1c, formation of $\mathrm{N}$ Mannich bases $(\mathbf{2 b}, \mathbf{2 c})$ and 5-methyl-4-thiazolidinone derivatives(4a) caused a decrease in antiviral activity against YFV. 5-Nonsubstituted 4-thiazolidinone derivative (3c) exhibited no change in activity when compared to 1c, but demonstrated higher toxicity, whereas compounds $\mathbf{2 b}, \mathbf{3 a}, \mathbf{3 f}, \mathbf{4 e}$ and $\mathbf{4 f}$ inhibited the growth of BVDV. The $\mathrm{EC}_{50}$ values of these compounds are 40, $50,44,13$ and $48 \mu \mathrm{g} / \mathrm{mL}$, respectively. The $\mathrm{EC}_{50}$ value of ribavirin is $40 \mu \mathrm{g} / \mathrm{mL}$ in the tests against BVDV. The results of $\mathbf{2 b}, \mathbf{3 a}, \mathbf{3 f}, \mathbf{4 e}$ and $\mathbf{4 f}$ were compared with ribavirin. Compound $\mathbf{4 e}$ showed much higher antiviral activity than that of ribavirin. Among 5-nitro-3-hydrazono-2indolinone derivatives tested against BVDV, 4-thiazolidinone derivatives $\mathbf{3}$ and $\mathbf{4}$ were more active than 3-thiosemicarbazones 1 and N-Mannich bases 2 . In addition, the presence of the methyl group at C-5 of the thiazolidinone ring also seems to have a significant impact on the resultant antiviral activity. Considering the anti-BVDV effect of substituents on the thiazolidinone ring, $\mathrm{R}_{1}$-aryl substituted were more active than the corresponding alkyl substituted ones. In fact, $\mathrm{R}_{1}$-(4-bromophenyl) substituted 5-methyl-4-thiazolidinone derivative 4e showed the most favourable antiviral activity against BVDV. 3-Hydrazono-5-nitro-2-indolinone derivatives 1-4 were also evaluated against the hepatitis $\mathrm{C}$ virus in Huh-5-2 cells, the parainfluenza-3 virus, reovirus-1, sindbis virus, coxsackie virus B4 and punta toro virus in vero cells, herpes simplex virus-1, herpes simplex virus-2, vaccinia virus, vesicular stomatitis virus in human embryonic lung (HEL) cells and vesicular stomatitis virus, coxsackie virus B4 and respiratory syncytial virus in HeLa cells. Whereas, no specific antiviral effects (i.e. minimal antivirally effective concentration $\geqslant 5$-fold lower than minimal cytotoxic concentration) were noted for any of the compounds against any of the viruses.

The obtained preliminary results suggest that some of these compounds might serve as potential candidates for antiviral agents. 
Table 1. Formulas, physical constants and elemental analyses for $\mathbf{3}$ and $\mathbf{4}$

\begin{tabular}{|c|c|c|c|c|c|c|c|c|}
\hline \multirow[t]{2}{*}{ Comp. } & \multirow[t]{2}{*}{$\mathrm{R}_{1}$} & \multirow[t]{2}{*}{$\mathrm{R}_{2}$} & \multirow{2}{*}{$\begin{array}{c}\text { Yield } \\
(\%)\end{array}$} & \multirow{2}{*}{$\begin{array}{c}\text { m.p. } \\
{ }^{\circ} \mathrm{C}\end{array}$} & \multirow{2}{*}{$\begin{array}{l}\text { Formula } \\
\text { (M.W.) }\end{array}$} & \multicolumn{3}{|c|}{ Analysis(calc./found) } \\
\hline & & & & & & $\mathrm{C}$ & $\mathrm{H}$ & $\mathrm{N}$ \\
\hline \multirow[t]{2}{*}{$\mathbf{3 a}$} & $\mathrm{CH}_{3}$ & $\mathrm{H}$ & 84 & $>300$ & $\mathrm{C}_{12} \mathrm{H}_{9} \mathrm{~N}_{5} \mathrm{O}_{4} \mathrm{~S} \cdot \mathrm{H}_{2} \mathrm{O}$ & 42.73 & 3.29 & 20.76 \\
\hline & & & & & $(337.31)$ & 43.13 & 3.53 & 21.13 \\
\hline \multirow[t]{2}{*}{$3 \mathbf{b}$} & $\mathrm{C}_{2} \mathrm{H}_{5}$ & $\mathrm{H}$ & 96 & $>300$ & $\mathrm{C}_{13} \mathrm{H}_{11} \mathrm{~N}_{5} \mathrm{O}_{4} \mathrm{~S} .1 / 2 \mathrm{H}_{2} \mathrm{O}$ & 45.61 & 3.53 & 20.45 \\
\hline & & & & & $(342.33)$ & 45.46 & 3.87 & 20.64 \\
\hline \multirow[t]{2}{*}{$3 \mathbf{c}$} & $\mathrm{CH}_{2}-\mathrm{CH}=\mathrm{CH}_{2}$ & $\mathrm{H}$ & 99 & $>300$ & $\mathrm{C}_{14} \mathrm{H}_{11} \mathrm{~N}_{5} \mathrm{O}_{4} \mathrm{~S}$ & 48.69 & 3.21 & 20.27 \\
\hline & & & & & $(345.33)$ & 48.43 & 3.67 & 19.71 \\
\hline \multirow[t]{2}{*}{ 3d } & $\mathrm{n}-\mathrm{C}_{4} \mathrm{H}_{9}$ & $\mathrm{H}$ & 99 & $>300$ & $\mathrm{C}_{15} \mathrm{H}_{15} \mathrm{~N}_{5} \mathrm{O}_{4} \mathrm{~S} .1 / 2 \mathrm{H}_{2} \mathrm{O}$ & 48.64 & 4.35 & 18.90 \\
\hline & & & & & $(370.39)$ & 48.63 & 4.40 & 18.58 \\
\hline \multirow[t]{2}{*}{$3 e$} & cycl- $\mathrm{C}_{6} \mathrm{H}_{11}$ & $\mathrm{H}$ & 95 & $>300$ & $\mathrm{C}_{17} \mathrm{H}_{17} \mathrm{~N}_{5} \mathrm{O}_{4} \mathrm{~S}$ & 52.70 & 4.42 & 18.07 \\
\hline & & & & & $(387.41)$ & 52.44 & 4.59 & 18.10 \\
\hline \multirow[t]{2}{*}{$3 f$} & $\mathrm{C}_{6} \mathrm{H}_{5}$ & $\mathrm{H}$ & 88 & $>300$ & $\mathrm{C}_{17} \mathrm{H}_{11} \mathrm{~N}_{5} \mathrm{O}_{4} \mathrm{~S}$ & 53.54 & 2.90 & 18.36 \\
\hline & & & & & (381.37) & 54.10 & 3.10 & 18.28 \\
\hline \multirow[t]{2}{*}{$3 g$} & $\mathrm{C}_{6} \mathrm{H}_{4} \mathrm{CH}_{3}(4-)$ & $\mathrm{H}$ & 99 & $>300$ & $\mathrm{C}_{18} \mathrm{H}_{13} \mathrm{~N}_{5} \mathrm{O}_{4} \mathrm{~S}$ & 54.68 & 3.31 & 17.71 \\
\hline & & & & & (395.39) & 54.82 & 3.35 & 17.84 \\
\hline \multirow[t]{2}{*}{$3 h$} & $\mathrm{C}_{6} \mathrm{H}_{4} \mathrm{Br}(4-)$ & $\mathrm{H}$ & 96 & $>300$ & $\mathrm{C}_{17} \mathrm{H}_{10} \mathrm{BrN}_{5} \mathrm{O}_{4} \mathrm{~S}$ & 44.36 & 2.19 & 15.21 \\
\hline & & & & & $(460.27)$ & 43.72 & 2.33 & 15.30 \\
\hline \multirow[t]{2}{*}{$3 \mathbf{i}$} & $\mathrm{C}_{6} \mathrm{H}_{4} \mathrm{Cl}(4-)$ & $\mathrm{H}$ & 85 & $>300$ & $\mathrm{C}_{17} \mathrm{H}_{10} \mathrm{ClN}_{5} \mathrm{O}_{4} \mathrm{~S}$ & 49.10 & 2.42 & 16.84 \\
\hline & & & & & $(415.81)$ & 49.11 & 2.44 & 16.87 \\
\hline \multirow[t]{2}{*}{$\mathbf{3 j}$} & $\mathrm{C}_{6} \mathrm{H}_{4} \mathrm{~F}(4-)$ & $\mathrm{H}$ & 92 & $>300$ & $\mathrm{C}_{17} \mathrm{H}_{10} \mathrm{FN}_{5} \mathrm{O}_{4} \mathrm{~S}$ & 51.12 & 2.52 & 17.53 \\
\hline & & & & & $(399.36)$ & 52.01 & 2.77 & 17.27 \\
\hline \multirow[t]{2}{*}{$4 a$} & $\mathrm{CH}_{2}-\mathrm{CH}=\mathrm{CH}_{2}$ & $\mathrm{CH}_{3}$ & 98 & $238-40$ & $\mathrm{C}_{15} \mathrm{H}_{13} \mathrm{~N}_{5} \mathrm{O}_{4} \mathrm{~S}$ & 50.13 & 3.64 & 19.48 \\
\hline & & & & & $(359.36)$ & 50.27 & 3.16 & 18.99 \\
\hline \multirow[t]{2}{*}{$4 b$} & $\mathrm{n}-\mathrm{C}_{4} \mathrm{H}_{9}$ & $\mathrm{CH}_{3}$ & 100 & $253-5$ & $\mathrm{C}_{16} \mathrm{H}_{17} \mathrm{~N}_{5} \mathrm{O}_{4} \mathrm{~S}$ & 51.19 & 4.56 & 18.65 \\
\hline & & & & & $(375.40)$ & 51.60 & 4.31 & 18.38 \\
\hline \multirow[t]{2}{*}{$4 c$} & $\mathrm{C}_{6} \mathrm{H}_{5}$ & $\mathrm{CH}_{3}$ & 94 & $>300$ & $\mathrm{C}_{18} \mathrm{H}_{13} \mathrm{~N}_{5} \mathrm{O}_{4} \mathrm{~S}$ & 54.68 & 3.31 & 17.71 \\
\hline & & & & & (395.39) & 55.13 & 3.08 & 17.53 \\
\hline \multirow[t]{2}{*}{$4 d$} & $\mathrm{C}_{6} \mathrm{H}_{4} \mathrm{CH}_{3}(4-)$ & $\mathrm{CH}_{3}$ & 77 & $282-3$ & $\mathrm{C}_{19} \mathrm{H}_{15} \mathrm{~N}_{5} \mathrm{O}_{4} \mathrm{~S}$ & 55.73 & 3.69 & 17.11 \\
\hline & & & & & $(409.42)$ & 55.80 & 3.31 & 17.03 \\
\hline \multirow[t]{2}{*}{$4 e$} & $\mathrm{C}_{6} \mathrm{H}_{4} \mathrm{Br}(4-)$ & $\mathrm{CH}_{3}$ & 91 & $>300$ & $\mathrm{C}_{18} \mathrm{H}_{12} \mathrm{BrN}_{5} \mathrm{O}_{4} \mathrm{~S}$ & 45.58 & 2.54 & 14.76 \\
\hline & & & & & $(474.29)$ & 46.01 & 2.11 & 14.21 \\
\hline \multirow[t]{2}{*}{$4 f$} & $\mathrm{C}_{6} \mathrm{H}_{4} \mathrm{Cl}(4-)$ & $\mathrm{CH}_{3}$ & 92 & $>300$ & $\mathrm{C}_{18} \mathrm{H}_{12} \mathrm{ClN}_{5} \mathrm{O}_{4} \mathrm{~S}$ & 50.29 & 2.81 & 16.29 \\
\hline & & & & & $(429.83)$ & 50.21 & 2.96 & 16.01 \\
\hline \multirow[t]{2}{*}{$4 g$} & $\mathrm{C}_{6} \mathrm{H}_{4} \mathrm{~F}(4-)$ & $\mathrm{CH}_{3}$ & 94 & $>300$ & $\mathrm{C}_{18} \mathrm{H}_{12} \mathrm{FN}_{5} \mathrm{O}_{4} \mathrm{~S}$ & 52.30 & 2.92 & 16.94 \\
\hline & & & & & $(413.38)$ & 52.67 & 2.55 & 16.56 \\
\hline \multirow[t]{2}{*}{$4 h$} & $\mathrm{C}_{6} \mathrm{H}_{4} \mathrm{NO}_{2}(4-)$ & $\mathrm{CH}_{3}$ & 88 & $>300$ & $\mathrm{C}_{18} \mathrm{H}_{12} \mathrm{~N}_{6} \mathrm{O}_{6} \mathrm{~S}$ & 49.09 & 2.74 & 19.08 \\
\hline & & & & & (440.39) & 48.90 & 2.56 & 18.61 \\
\hline
\end{tabular}


Table 2. Antiviral activity and cytotoxicity on YFV and BVDV of compounds 1-4

\begin{tabular}{|c|c|c|c|c|c|c|c|}
\hline \multirow[b]{2}{*}{ Comp. } & \multirow[b]{2}{*}{$\mathrm{R}_{1}$} & \multirow[b]{2}{*}{$\mathrm{R}_{2}$} & \multirow[b]{2}{*}{$\mathrm{X}$} & \multicolumn{2}{|c|}{ YFV (Vero) } & \multicolumn{2}{|c|}{ BVDV (MDBK) } \\
\hline & & & & $\begin{array}{c}\mathrm{EC}_{50} \\
(\mu \mathrm{g} / \mathrm{mL})\end{array}$ & $\begin{array}{c}\text { MTC } \\
(\mu \mathrm{g} / \mathrm{mL})\end{array}$ & $\begin{array}{c}\mathrm{EC}_{50} \\
(\mu \mathrm{g} / \mathrm{mL})\end{array}$ & $\begin{array}{c}\mathrm{CC}_{50} \\
(\mu \mathrm{g} / \mathrm{mL})\end{array}$ \\
\hline $1 \mathbf{a}$ & $\mathrm{CH}_{3}$ & - & - & $>100$ & 100 & $>50$ & $>50$ \\
\hline $1 b$ & $\mathrm{C}_{2} \mathrm{H}_{5}$ & - & - & 1.96 & 20 & $>50$ & $>50$ \\
\hline 1c & $\mathrm{CH}_{2}-\mathrm{CH}=\mathrm{CH}_{2}$ & - & - & 2.18 & 100 & $>50$ & $>50$ \\
\hline 1d & $\mathrm{n}-\mathrm{C}_{4} \mathrm{H}_{9}$ & - & - & $<0.8$ & 20 & $>50$ & $>50$ \\
\hline $1 \mathrm{e}$ & cycl- $\mathrm{C}_{6} \mathrm{H}_{11}$ & - & - & $<0.8$ & 20 & $>5$ & 5 \\
\hline 1f & $\mathrm{C}_{6} \mathrm{H}_{5}$ & - & - & $<0.8$ & 4 & $>50$ & $>50$ \\
\hline $1 \mathrm{~g}$ & $\mathrm{C}_{6} \mathrm{H}_{4} \mathrm{CH}_{3}(4-)$ & - & - & 1.96 & 20 & $>17$ & 17 \\
\hline $1 \mathrm{~h}$ & $\mathrm{C}_{6} \mathrm{H}_{4} \mathrm{Br}(4-)$ & - & - & $<0.8$ & 20 & $>17$ & 17 \\
\hline $1 \mathbf{i}$ & $\mathrm{C}_{6} \mathrm{H}_{4} \mathrm{Cl}(4-)$ & - & - & $<0.8$ & 4 & $>19$ & 19 \\
\hline $\mathbf{1 j}$ & $\mathrm{C}_{6} \mathrm{H}_{4} \mathrm{~F}(4-)$ & - & - & $<0.8$ & 4 & $>20$ & 20 \\
\hline $1 \mathrm{k}$ & $\mathrm{C}_{6} \mathrm{H}_{4} \mathrm{NO}_{2}(4-)$ & - & - & 4 & 20 & $>22$ & 22 \\
\hline $2 \mathbf{a}$ & $\mathrm{CH}_{3}$ & - & $\mathrm{O}$ & $>100$ & 100 & $>20$ & 20 \\
\hline $2 \mathrm{~b}$ & $\mathrm{CH}_{2}-\mathrm{CH}=\mathrm{CH}_{2}$ & - & $\mathrm{O}$ & 11 & 100 & 40 & $>\mathbf{5 0}$ \\
\hline $2 c$ & $\mathrm{CH}_{2}-\mathrm{CH}=\mathrm{CH}_{2}$ & - & $\mathrm{CH}_{2}$ & $>4$ & 4 & $>16$ & 16 \\
\hline 2d & $\mathrm{n}-\mathrm{C}_{4} \mathrm{H}_{9}$ & - & $\mathrm{O}$ & $>20$ & 20 & $>19$ & 19 \\
\hline $2 \mathrm{e}$ & cycl- $\mathrm{C}_{6} \mathrm{H}_{11}$ & - & $\mathrm{O}$ & $<0.8$ & 20 & $>17$ & 17 \\
\hline $2 f$ & cycl- $\mathrm{C}_{6} \mathrm{H}_{11}$ & - & $\mathrm{CH}_{2}$ & $<0.8$ & 20 & $>21$ & 21 \\
\hline $2 g$ & $\mathrm{C}_{6} \mathrm{H}_{5}$ & - & $\mathrm{O}$ & 2.18 & 20 & $>50$ & $>50$ \\
\hline $2 \mathrm{~h}$ & $\mathrm{C}_{6} \mathrm{H}_{5}$ & - & $\mathrm{CH}_{2}$ & $>20$ & 20 & $>20$ & 20 \\
\hline $2 \mathbf{i}$ & $\mathrm{C}_{6} \mathrm{H}_{4} \mathrm{CH}_{3}(4-)$ & - & $\mathrm{O}$ & 3.06 & 20 & $>21$ & 21 \\
\hline $2 j$ & $\mathrm{C}_{6} \mathrm{H}_{4} \mathrm{CH}_{3}(4-)$ & - & $\mathrm{CH}_{2}$ & 1.59 & 20 & $>19$ & 19 \\
\hline $2 \mathrm{k}$ & $\mathrm{C}_{6} \mathrm{H}_{4} \mathrm{Cl}(4-)$ & - & $\mathrm{O}$ & $>20$ & 20 & $>17$ & 17 \\
\hline 21 & $\mathrm{C}_{6} \mathrm{H}_{4} \mathrm{Cl}(4-)$ & - & $\mathrm{CH}_{2}$ & $>20$ & 20 & $>19$ & 19 \\
\hline $3 \mathbf{a}$ & $\mathrm{CH}_{3}$ & $\mathrm{H}$ & - & $>20$ & 20 & 50 & $>\mathbf{5 0}$ \\
\hline $3 \mathbf{b}$ & $\mathrm{C}_{2} \mathrm{H}_{5}$ & $\mathrm{H}$ & - & 2.52 & 100 & $>17$ & 17 \\
\hline $3 c$ & $\mathrm{CH}_{2}-\mathrm{CH}=\mathrm{CH}_{2}$ & $\mathrm{H}$ & - & 2.18 & 20 & $>4$ & 4 \\
\hline 3d & $\mathrm{n}-\mathrm{C}_{4} \mathrm{H}_{9}$ & $\mathrm{H}$ & - & $>100$ & 100 & $>50$ & $>50$ \\
\hline $3 e$ & cycl- $\mathrm{C}_{6} \mathrm{H}_{11}$ & $\mathrm{H}$ & - & 1.96 & 20 & $>3$ & 3 \\
\hline $3 f$ & $\mathrm{C}_{6} \mathrm{H}_{5}$ & $\mathrm{H}$ & - & $>20$ & 20 & 44 & $>\mathbf{5 0}$ \\
\hline $3 g$ & $\mathrm{C}_{6} \mathrm{H}_{4} \mathrm{CH}_{3}(4-)$ & $\mathrm{H}$ & - & 4 & 20 & $>50$ & $>50$ \\
\hline $3 h$ & $\mathrm{C}_{6} \mathrm{H}_{4} \mathrm{Br}(4-)$ & $\mathrm{H}$ & - & $>20$ & 20 & $>5$ & 5 \\
\hline $3 \mathbf{i}$ & $\mathrm{C}_{6} \mathrm{H}_{4} \mathrm{Cl}(4-)$ & $\mathrm{H}$ & - & 1.52 & 20 & $>2$ & 2 \\
\hline $3 \mathbf{j}$ & $\mathrm{C}_{6} \mathrm{H}_{4} \mathrm{~F}(4-)$ & $\mathrm{H}$ & - & $>20$ & 20 & $>9$ & 9 \\
\hline $4 a$ & $\mathrm{CH}_{2}-\mathrm{CH}=\mathrm{CH}_{2}$ & $\mathrm{CH}_{3}$ & - & $>20$ & 20 & $>50$ & $>50$ \\
\hline $4 b$ & $\mathrm{n}-\mathrm{C}_{4} \mathrm{H}_{9}$ & $\mathrm{CH}_{3}$ & - & $>20$ & 20 & $>50$ & $>50$ \\
\hline $4 c$ & $\mathrm{C}_{6} \mathrm{H}_{5}$ & $\mathrm{CH}_{3}$ & - & $>20$ & 20 & $>7$ & 7 \\
\hline 4d & $\mathrm{C}_{6} \mathrm{H}_{4} \mathrm{CH}_{3}(4-)$ & $\mathrm{CH}_{3}$ & - & $>20$ & 20 & $>41$ & 41 \\
\hline $4 e$ & $\mathrm{C}_{6} \mathrm{H}_{4} \mathrm{Br}(4-)$ & $\mathrm{CH}_{3}$ & - & 1.96 & 4 & 13 & $>\mathbf{5 0}$ \\
\hline $4 f$ & $\mathrm{C}_{6} \mathrm{H}_{4} \mathrm{Cl}(4-)$ & $\mathrm{CH}_{3}$ & - & 1.59 & 20 & 48 & $>\mathbf{5 0}$ \\
\hline $4 \mathrm{~g}$ & $\mathrm{C}_{6} \mathrm{H}_{4} \mathrm{~F}(4-)$ & $\mathrm{CH}_{3}$ & - & $>4$ & 4 & $>3$ & 3 \\
\hline $4 h$ & $\mathrm{C}_{6} \mathrm{H}_{4} \mathrm{NO}_{2}(4-)$ & $\mathrm{CH}_{3}$ & - & $>100$ & 100 & $>18$ & 18 \\
\hline Rib. & & & & 45 & $>100$ & 40 & \\
\hline
\end{tabular}




\section{Experimental Section}

General Procedures. M.p.'s were determined on a Büchi 530 apparatus and are uncorrected. Elemental analyses were performed on Carlo Erba 1106 elemental analyzers. IR spectra were recorded on $\mathrm{KBr}$ discs, using a Perkin Elmer 1600 FT-IR spectrometer. ${ }^{1} \mathrm{H}$ NMR and ${ }^{13} \mathrm{C}$ NMR spectra were obtained on Bruker AC 200 (200 and 50.3 MHz) spectrophotometer using DMSO$\mathrm{d}_{6}$. EIMS $(70 \mathrm{eV})$ were recorded onVG Zab Spec.

Synthesis of 5-nitro-3-[(5-nonsubstituted/methyl-4-thiazolidinone-2-ylidene)hydrazono]$\mathbf{1 H}$-2-indolinones (3a-j and $\mathbf{4 a - h})$. To a suspension of an appropriate thiosemicarbazone $\mathbf{1}$ (2.5 mmol) in absolute ethanol $(25 \mathrm{~mL})$, anhydrous sodium acetate $(10 \mathrm{mmol})$ and ethyl bromoacetate or ethyl 2-bromopropionate $(2.5 \mathrm{mmol})$ were added. The reaction mixture was refluxed for $4 \mathrm{~h}$, cooled, diluted with water and allowed to stand overnight. The yellow crystals obtained were filtered and purified by re-crystallization from ethanol.

3-[(3-n-Butyl-4-thiazolidinone-2-ylidene)hydrazono]-5-nitro-1H-2-indolinone (3d). IR $\left(v, \mathrm{~cm}^{-1}\right): 3208(\mathrm{NH}), 1734,1689(\mathrm{C}=\mathrm{O}), 1496,1339\left(\mathrm{NO}_{2}\right) .{ }^{1} \mathrm{H}$ NMR $(\delta, \mathrm{ppm}): 0.94(\mathrm{t}, J=7.2$ $\mathrm{Hz}, 3 \mathrm{H}, \mathrm{CH}_{3}$ ), 1.47 (m, 2H, butyl), 1.75 (quin, $J=7.4 \mathrm{~Hz}, 2 \mathrm{H}$, butyl), 3.90 (t, J=7.3 Hz, 2H, butyl), 4.09, $4.11\left(2 \mathrm{~s}, 2 \mathrm{H}, \mathrm{SCH}_{2}\right), 7.07$ (d, $J=8.6 \mathrm{~Hz}, 1 \mathrm{H}$, indole $\left.\mathrm{C}_{7}-\mathrm{H}\right), 8.29$ (dd, J=8.7, $2.3 \mathrm{~Hz}$, $1 \mathrm{H}$, indole $\left.\mathrm{C}_{6}-\mathrm{H}\right), 8.94\left(\mathrm{~d}, \mathrm{~J}=2.3 \mathrm{~Hz}, 1 \mathrm{H}\right.$, indole $\left.\mathrm{C}_{4}-\mathrm{H}\right), 11.34(\mathrm{~s}, 1 \mathrm{H}$, indole $\mathrm{NH})$. EIMS (m/z, \%): $361\left(\mathrm{M}^{+}, 4\right), 331$ (17), 277 (14), 191 (9), 171 (14), 156 (40), 133 (19), 117 (57), 78 (86), 63 $(100)$.

5-Nitro-3-[(3-phenyl-4-thiazolidinone-2-ylidene)hydrazono]-1H-2-indolinone (3f). IR $\left(v, \mathrm{~cm}^{-1}\right): 3320(\mathrm{NH}), 1735,1698(\mathrm{C}=\mathrm{O}), 1524,1341\left(\mathrm{NO}_{2}\right) .{ }^{1} \mathrm{H}$ NMR $(\delta, \mathrm{ppm}): 4.20,4.24$ $\left(2 \mathrm{~s}, 2 \mathrm{H}, \mathrm{SCH}_{2}\right), 6.99\left(\mathrm{~d}, J=8.4 \mathrm{~Hz}, 1 \mathrm{H}\right.$, indole $\left.\mathrm{C}_{7}-\mathrm{H}\right), 7.42-8.20\left(\mathrm{~m}, 7 \mathrm{H}\right.$, indole $\mathrm{C}_{4,6}-\mathrm{H}$ and phenyl), 11.28 (br.s, 1H, indole NH). EIMS (m/z, \%): $381\left(\mathrm{M}^{+}, 100\right), 351$ (26), 281 (13), 191 (88), 190 (15), 135 (42), 118 (37), 77 (36).

3-[(3-(4-Chlorophenyl)-4-thiazolidinone-2-ylidene)hydrazono]-5-nitro-1H-2-indolinone (3i). IR $\left(v, \mathrm{~cm}^{-1}\right): 3296(\mathrm{NH}), 1734,1705(\mathrm{C}=\mathrm{O}), 1493,1334\left(\mathrm{NO}_{2}\right) .{ }^{1} \mathrm{H}$ NMR $(\delta, \mathrm{ppm}): 4.19,4.22$ $\left(2 \mathrm{~s}, 2 \mathrm{H}, \mathrm{SCH}_{2}\right), 7.00\left(\mathrm{~d}, J=8.4 \mathrm{~Hz}, 1 \mathrm{H}\right.$, indole $\left.\mathrm{C}_{7}-\mathrm{H}\right), 7.52-8.22\left(\mathrm{~m}, 6 \mathrm{H}\right.$, indole $\mathrm{C}_{4,6}-\mathrm{H}$ and phenyl), 11.30 (br.s, 1H, indole NH). EIMS (m/z, \%): 415 [M+1, $100(417,39)], 387$ [33 (389, 8)], 225 [33 (227, 13)], 191 (9), 176 (21), 169 (30), 152 [26 (154, 9)], 132 (20), 118 (9).

3-[(3-Allyl-5-methyl-4-thiazolidinone-2-ylidene)hydrazono]-5-nitro-1H-2-indolinone (4a). IR $\left(v, \mathrm{~cm}^{-1}\right): 3179(\mathrm{NH}), 1734,1698(\mathrm{C}=\mathrm{O}), 1505,1339\left(\mathrm{NO}_{2}\right) .{ }^{1} \mathrm{H}$ NMR $(\delta, \mathrm{ppm}): 1.60(\mathrm{~d}$, $\left.J=7.3 \mathrm{~Hz}, 3 \mathrm{H}, \mathrm{CH}_{3}\right), 4.47$ (q, J=7.3 Hz, 1H, SCH), 4.52 (d, J=4.8 Hz, 2H, $\mathrm{CH}_{2}-\mathrm{CH}=\mathrm{CH}_{2}$ ), 5.23, $5.33\left(\mathrm{dd}, J=17.5,10.4 \mathrm{~Hz}, 2 \mathrm{H}, \mathrm{CH}_{2}-\mathrm{CH}=\mathrm{CH}_{2}\right), 5.88-6.07\left(\mathrm{~m}, 1 \mathrm{H}, \mathrm{CH}_{2}-\mathrm{CH}=\mathrm{CH}_{2}\right), 7.06(\mathrm{~d}, J=8.7$ $\mathrm{Hz}, 1 \mathrm{H}$, indole $\left.\mathrm{C}_{7}-\mathrm{H}\right), 8.30\left(\mathrm{dd}, J=8.7,2.3 \mathrm{~Hz}, 1 \mathrm{H}\right.$, indole $\left.\mathrm{C}_{6}-\mathrm{H}\right), 8.91(\mathrm{~d}, J=2.3 \mathrm{~Hz}, 1 \mathrm{H}$, indole $\left.\mathrm{C}_{4}-\mathrm{H}\right), 11.43$ (s, 1H, indole $\left.\mathrm{NH}\right)$.

5-Nitro-3-[(3-phenyl-5-methyl-4-thiazolidinone-2-ylidene)hydrazono]-1H-2-indolinone (4c). IR $\left(v, \mathrm{~cm}^{-1}\right): 3296(\mathrm{NH}), 1756,1699(\mathrm{C}=\mathrm{O}), 1519,1350\left(\mathrm{NO}_{2}\right) .{ }^{1} \mathrm{H}$ NMR $(\delta, \mathrm{ppm}): 1.70(\mathrm{~d}$, $\left.J=7.2 \mathrm{~Hz}, 3 \mathrm{H}, \mathrm{CH}_{3}\right), 4.55$ (q, J=7.2 Hz, 1H, SCH), 6.99 (d, J=8.6 Hz, 1H, indole $\left.\mathrm{C}_{7}-\mathrm{H}\right), 7.50$ 7,66 (m, 5H, ar), 8.14-8.17 (m, $1 \mathrm{H}$, indole $\left.\mathrm{C}_{6}-\mathrm{H}\right), 8.21\left(\mathrm{~d}, J=2.5 \mathrm{~Hz}, 1 \mathrm{H}\right.$, indole $\left.\mathrm{C}_{4}-\mathrm{H}\right), 11.31(\mathrm{~s}$, 
$1 \mathrm{H}$, indole $\mathrm{NH}) .{ }^{13} \mathrm{C}$ NMR ( $\delta$, proton decoupled): 19.30 (thiazolidin $\mathrm{CH}_{3}$ ), 42.05 (thiazolidin $\mathrm{C}_{5}$ ), 110.55 (indole $\mathrm{C}_{7}$ ), 116.00 (indole $\mathrm{C}_{3 \mathrm{a}}$ ), 122.88 (indole $\mathrm{C}_{6}$ ), 127.59 (phenyl $\mathrm{C}_{2,6}$ ), 128.78 (indole $\mathrm{C}_{4}$ ), 129.16 (phenyl $\mathrm{C}_{4}$ ), 129.32 (phenyl $\mathrm{C}_{3,5}$ ), 135.00 (phenyl $\mathrm{C}_{1}$ ), 142.00 (indole $\mathrm{C}_{5,7 \mathrm{a}}$ ), 149.00 (indole $\mathrm{C}_{3}$ ), 154.00 (thiazolidin $\mathrm{C}_{2}$ ), 165.00 (indole $\mathrm{C}=\mathrm{O}$ ), 175.11 (thiazolidin $\mathrm{C}=\mathrm{O}$ ). EIMS $(\mathrm{m} / \mathrm{z}$, \%): $395\left(\mathrm{M}^{+}, 100\right), 320$ (52), 205 (86), 190 (6), 135 (48), 118 (36).

\section{3-[(3-(4-Bromophenyl)-5-methyl-4-thiazolidinone-2-ylidene)hydrazono]-5-nitro-1H-2-}

indolinone (4e). IR $\left(v, \mathrm{~cm}^{-1}\right): 3273 \mathrm{~cm}^{-1}(\mathrm{NH}), 1750,1691(\mathrm{C}=\mathrm{O}), 1490,1328\left(\mathrm{NO}_{2}\right) .{ }^{1} \mathrm{H}$ NMR

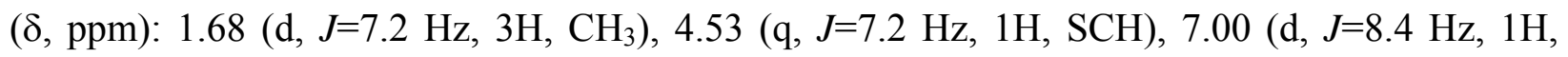
indole $\left.\mathrm{C}_{7}-\mathrm{H}\right), 7.51(\mathrm{~d}, J=8.6 \mathrm{~Hz}, 2 \mathrm{H}, \mathrm{ar}), 7.80(\mathrm{~d}, J=8.7 \mathrm{~Hz}, 2 \mathrm{H}, \mathrm{ar}), 8.16-8.20\left(\mathrm{~m}, 1 \mathrm{H}\right.$, indole $\mathrm{C}_{6-}$ $\mathrm{H}), 8.23\left(\mathrm{~d}, J=2.5 \mathrm{~Hz}, 1 \mathrm{H}\right.$, indole $\left.\mathrm{C}_{4}-\mathrm{H}\right), 11.38(\mathrm{~s}, 1 \mathrm{H}$, indole $\mathrm{NH})$. EIMS (m/z, \%): $473\left[\mathrm{M}^{+}, 23\right.$ $(475,23)], 395$ (96), 283 [16 (285, 17)], 205 (100), 192 (17), 135 (52), 118 (48).

5-Nitro-3-[(3-(4-nitrophenyl)-5-methyl-4-thiazolidinone-2-ylidene)hydrazono]-1H-2indolinone (4h). IR $\left(\mathrm{v}, \mathrm{cm}^{-1}\right): 3314 \mathrm{~cm}^{-1}(\mathrm{NH}), 1747,1696(\mathrm{C}=\mathrm{O}), 1516,1349\left(\mathrm{NO}_{2}\right) .{ }^{1} \mathrm{H}$ NMR

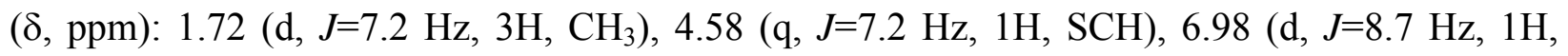
indole $\left.\mathrm{C}_{7}-\mathrm{H}\right), 7.88\left(\mathrm{~d}, J=9.1 \mathrm{~Hz}, 2 \mathrm{H}\right.$, ar), $7.98\left(\mathrm{~d}, J=2.4 \mathrm{~Hz}, 1 \mathrm{H}\right.$, indole $\left.\mathrm{C}_{4}-\mathrm{H}\right), 8.18$ (dd, $J=8.7$, $2.6 \mathrm{~Hz}, 1 \mathrm{H}$, indole $\left.\mathrm{C}_{6}-\mathrm{H}\right), 8.49(\mathrm{~d}, J=8.9 \mathrm{~Hz}, 2 \mathrm{H}$, ar), $11.38(\mathrm{~s}, 1 \mathrm{H}$, indole $\mathrm{NH})$.

Antiviral activity assays. Antiviral activity against the yellow fever virus (YFV), hepatitis $\mathrm{C}$ virus (HCV), the parainfluenza-3 virus, reovirus-1, sindbis virus, coxsackie virus B4, punta toro virus, herpes simplex virus-1, herpes simplex virus-2, vaccinia virus, vesicular stomatitis virus, coxsackie virus B4 and respiratory syncytial virus was determined essentially as described previously $^{11}$.

Antiviral activity assay for BVDV. MDBK cells were seeded at a density of $5 \times 10^{3}$ per well in 96-well cell culture plates in MEM-FCS. Following $24 \mathrm{~h}$ incubation at $37^{\circ} \mathrm{C}$ and $5 \% \mathrm{CO}_{2}$ medium was removed and 5-fold serial dilutions of the test compounds were added in a total volume of $100 \mu \mathrm{L}$, after which the cp BVDV virus inoculum [100 CCID $_{50}(50 \%$ cell culture infective dose) in $100 \mu \mathrm{L}$ ] was added to each well. This inoculum resulted in a greater than $90 \%$ destruction of the cell monolayer after 4-5 days of incubation at $37^{\circ} \mathrm{C}$. Uninfected cells and cells receiving virus without compound were included in each assay plate. After 5 days, medium was removed, and $90 \mu \mathrm{L}$ of MEM-FCS supplemented with $10 \mu \mathrm{L}$ of MTS/PMS solution (Promega, Leiden, The Netherlands) was added to each well. Following a $2 \mathrm{~h}$ incubation period at $37^{\circ} \mathrm{C}$, the optical density of each well was read at $498 \mathrm{~nm}$ in a microplate reader. The $50 \%$ effective concentration $\left(\mathrm{EC}_{50}\right)$ was defined as the concentration of compound of which $50 \%$ cell viability was protected from virus-induced cytopathic effect $(\mathrm{CPE})^{4}$.

Cytostatic assay for BVDV. MDBK cells were seeded at a density of $5 \times 10^{3}$ cells per well of a 96-well plate in MEM-FCS. 24 hours later, serial dilutions of the test compounds were added. Cells were allowed to proliferate for 3 days at $37^{\circ} \mathrm{C}$, after which the cell number was determined by means of the MTS/PMS method (Promega). The $50 \%$ cytotoxic concentration $\left(\mathrm{CC}_{50}\right)$ was defined as the concentration that inhibited the proliferation of exponentially growing cells by $50 \%$. 


\section{Acknowledgements}

This work was supported by Istanbul University Research Fund. Project Numbers: Ö-720/ 081299, Ö-780/ 26042000 and Ö-1097/ 15102001.

\section{References}

1. (a) Teitz, Y.; Barko, N.; Abramoff, M.; Ronen, D.; Chemotherapy 1994, 40, 195. (b) Teitz, Y.; Ronen, D.; Vansover, A.; Stematsky, T.; Riggs, J. L. Antiviral Res. 1994, 24, 305. (c) Winter, J.; Hall, R.L.; Moyer, R.W. Virology 1995, 211, 462.

2. Sethi, M. L. Foye's Principles of Medicinal Chemistry, Lippincott Williams and Wilkins: Philadelphia, 2002, chapter 39.

3. (a) Zhang, H.; Vrang, L.; Bacbro, K.; Lind, P.; Sahlberg, C.; Unge, T.; Öberg, B. Antiviral Res. 1995, 28, 331. (b) Mishra, V.; Pandeya, S. N.; DeClercq, E.; Pannecouque, C.; Witvrouw, M. Pharm. Acta Helv. 1998, 73, 215.

4. Sun, J.-H.; Lemm, J. A.; O’Boyle, D. R.; Colonno, R.; Gao, M. J. Virology 2003, 77, 6753.

5. (a) Rao, A.; Carbone, A.; Chimirri, A.; De Clercq, E.; Monforte, A. M.; Monforte, P.; Pannecouque, C.; Zappala, M. Il Farmaco 2003, 58, 115. (b) Barreca, M. L.; Chimirri, A.; De Clercq, E.; De Luca, L.; Monforte, A. M.; Monforte, P.; Rao, A.; Zappalà, M. Il Farmaco 2003, 58, 259. (c) Rao, A.; Balzarini, J.; Carbone, A.; Chimirri, A.; De Clercq, E.; Monforte, A. M.; Monforte, P.; Pannecouque, C.; Zappalà, M. Il Farmaco 2004, 59, 33. (d) Rao, A.; Balzarini, J.; Carbone, A.; Chimirri, A.; De Clercq, E.; Monforte, A. M.; Monforte, P.; Pannecouque, C.; Zappalà, M. Antiviral Res. 2004, 63, 79.

6. (a) Petrov, I.; Grupce, O.; Stafilov, T. J. Mol. Struct. 1986, 142, 275. (b) Naumov, P.; Anastasova, F. Spectr. Acta 2001, 57A, 469. (c) Moustafa, M. A.; Bayomi, S. M.; El-Emam, A. A.; El-Kerdawy, M. M. Sci. Pharm. 1989, 57, 125.

7. Farghaly, A. M.; Habib, N. S.; Khalil, M. A.; El-Sayed, O. A.; Bistawroos, A. E. Arch. Pharm. (Weinheim) 1990, 323, 247.

8. (a) Omar, A.-M. M. E.; Eshba, N. H.; Salama, H. M. Arch Pharm. (Weinheim) 1984, 317, 701. (b) Varma, R. S.; Singh, A. P.; Singh, S. P. Org. Mass Spectr. 1992, 27, 17.

9. Karalı, N. Eur. J. Med. Chem. 2002, 37, 909.

10. Balzarini, J.; De Clercq, E.; Van Hemel, J.; Neyts, J.; Peys, E.; Ruttens, B.; Van der Eycken, J.; Vandenkerckhove, J. US Patent Appl. 20040209823, 514023000 (USPTO), A61K031/70 (Intl Class), 10/21/04; Davis, Brown, Koehn, Shors \& Roberts, P.C. The Financial Center - Des Moines, IA, US.

11. (a) Leysen, P.; De Clercq, E.; Neyts, J.; Clin. Microbiol. Rev. 2000, 13, 67. (b) De Clercq, E.; Holy, A.; Rosenberg, I.; Sakuma, T.; Balzarini, J.; Maudgal, P. C. Nature 1986, 323, 
464. (c) Balzarini, J.; Naesens, L.; Slachmuylders, J.; Niphuis, H.; Rosenberg, I.; Holy, A.; Schellekens, H.; De Clercq, E. AIDS 1991, 5, 21. (d) Neyts, J.; Meerbach, A.; McKenna, P.; De Clercq, E. Antiviral Res. 1996, 30, 125. 